\title{
HACIA LA DIAGNOSIS Y RECUPERACIÓN DE SENSORES ROBÓTICOS A BAJO NIVEL MEDIANTE INFERENCIA EN REDES BAYESIANAS
}

\author{
Manuel Castellano Quero, Juan Antonio Fernández Madrigal, Alfonso José García Cerezo \\ Departamento de Ingeniería de Sistemas y Automática, Universidad de Málaga \\ e-mail: $\{$ mcastellano, jafernandez, ajgarcia\}@uma.es
}

\section{Resumen}

Las implementaciones existentes en la actualidad sobre sensores virtuales no emplean un marco común matemáticamente riguroso. Por ello, en este trabajo tenemos como objetivo homogeneizar el soporte teórico de los sensores virtuales a bajo nivel, es decir, tratando directamente sus datos en bruto, de tal modo que puedan ser empleados en diagnóstico de fallos, recuperación de datos y otras funcionalidades sin cambiar el paradigma de base. La inferencia bayesiana constituye una manera genérica y rigurosa de abordar este problema; además, nos permite integrar conocimiento procedente de diversas fuentes (los propios dispositivos sensoriales, sentido común humano, datos del entorno, etc.) y se puede hibridar con otras metodologías como las redes neuronales o la lógica borrosa. Dado que el potencial de esta solución es considerablemente amplio, nos centramos aquí en el diagnóstico de averías, recuperación de datos y funcionalidades de integración de conocimiento externo. Nuestros resultados con un robot móvil real equipado con dos sensores de proximidad y con otros dispositivos más simples, demuestran que este marco tiene muchas posibilidades de mejorar el sistema sensorial de un robot por medio de técnicas de razonamiento de alto nivel.

Palabras clave: inferencia bayesiana, detección y recuperación de fallos, sensores virtuales

\section{INTRODUCCIÓN}

Los algoritmos de inferencia bayesiana son métodos matemáticos que permiten obtener nuevo conocimiento a partir del disponible en situaciones de incertidumbre [1]. Estos métodos tienen importantes ventajas en comparación con otras técnicas de inferencia; proporcionan información sobre la incertidumbre en los resultados, están basados en una teoría matemática rigurosa que explica la deducción, se puede hibridar con otras herramientas, puede integrar conocimiento experto e información procedente de fuentes heterogéneas y permite inferir el estado de una variable y el de sus causantes (normalmente denominados padres). El principal inconveniente es el coste computacional en términos de tiempo y memoria tanto de los algoritmos exactos como de los aproximados [1].

En Robótica, algunas de sus aplicaciones pueden encontrarse en localización y mapeado [2], control y tareas de manipulación [3], fusión de datos sensoriales para robots autónomos [4], programación lógica [5], etc. En este artículo nos centramos en el diagnóstico para robots móviles, en los que los sensores pueden fallar debido a numerosas razones (ruido, valores fuera de rango, malas condiciones ambientales, desconexiones, etc.). A pesar de que la inferencia bayesiana ha sido ampliamente utilizada en diagnóstico de fallos genérico [6], la mayor parte de los trabajos sobre diagnóstico sensorial en robots móviles emplean otras metodologías que no proporcionan las ventajas mencionadas anteriormente: técnicas analíticas, métodos heurísticos, redes neuronales, lógica borrosa, razonamiento basado en casos (CBR), etc. En general, el diagnóstico de sensores y la recuperación pueden considerarse situados en el contexto de los sensores virtuales o software, pero la investigación en este área es aún limitada [7], las soluciones existentes no tienen capacidades de inferencia especialmente destacables [8] y no están implementadas en un marco matemático riguroso [9].

En este trabajo proponemos redes bayesianas para mejorar la robustez sensorial. Nuestra propuesta tiene en cuenta las características de los sensores físicos del robot, pero también puede integrar otros tipos de conocimiento. En un trabajo previo [10] presentamos una versión preliminar de esta solución implementada para sensores muy sencillos: colisión, detectores de precipicio, detectores de levantamiento de rueda, codificadores rotatorios y giroscopios, y concluimos que el sistema podía alcanzar un rendimiento adecuado incluso con el empleo de algoritmos exactos de inferencia bayesiana. En este trabajo relajamos los requisitos computacionales e incluimos información sensorial más compleja: un telémetro láser 2D y, parcialmente, una cámara RGB-D. También integramos otros tipos de información no procedente de los sensores, como la del entorno. Nuestra red bayesiana es creada ahora de forma semi-automáti- 
ca, procedimental, dependiendo de la resolución de los datos que se requiera. En el estado actual de nuestra investigación, todas las variables de la red son discretas con un número finito de estados. Además, empleamos redes bayesianas estáticas; el estudio de su comportamiento en tiempo real no es nuestro propósito aquí.

Los resultados de varios experimentos muestran que un ordenador portátil convencional puede tratar redes bayesianas razonablemente grandes que sean capaces de detectar anomalías como los fallos de fuera de rango, condiciones ambientales severas, discontinuidades en las medidas, objetos indetectables (demasiado delgados, negros o transparentes), etc. Además, podemos identificar la causa más probable de las anomalias y los valores erróneos detectados pueden ser reemplazados por los correctos mediante inferencia probabilística sobre sensores alternativos, o teniendo en cuenta información adicional (externa al sistema sensorial).

El artículo se estructura como se indica a continuación. En la sección 2 se resume nuestra propuesta de sensor bayesiano. La seción 3 describe la implementación de una arquitectura sensorial para un robot móvil concreto equipado con los sensores antes mencionados. La sección 4 presenta los resultados obtenidos en los experimentos y, finalmente, la sección 5 resume las principales conclusiones de este estudio y propone futuras investigaciones.

\section{REDES BAYESIANAS PARA DIAGNÓSTICO SENSORIAL}

En primer lugar presentamos una breve definición de red bayesiana y citamos los principales algoritmos de inferencia. Para una revisión en mayor profundidad se recomienda [1] [11].

Una red bayesiana definida sobre un conjunto de variables $\mathbf{V}$ es una pareja $(G, \Theta)$ formada por un grafo acíclico dirigido, $\mathrm{G}$, sobre $\mathbf{V}$, denominado estructura de la red, y un conjunto de Tablas de Probabilidad Condicionada (CPTs), $\Theta$, para cada variable de $\mathbf{V}$, llamado parametrización de la red. La estructura del grafo representa las relaciones causales entre las variables mediante arcos, que indican relaciones de dependencia, mientras que las CPTs definen distribuciones de probabilidad sobre las variables.

En este caso estamos interesados en inferir nuevo conocimiento a partir del disponible, es decir, en deducir una distribución de probabilidad sobre un conjunto de nodos de consulta $\mathbf{Q}$ dados algunos nodos de evidencia $\mathbf{E}$, en definitiva, en obtener $\mathrm{P}(\mathbf{Q} \mid \mathbf{E})$. Esto se puede hacer mediante la aplicación repetida de teoría básica de la probabi- lidad (la regla de la cadena, el teorema de Bayes, etc.), aunque esto puede ser prohibitivo incluso para problemas de pequeña escala. Por tanto es necesario utilizar un algoritmo de inferencia bayesiana, para reducir la complejidad computacional.

Existen numeroros algoritmos de inferencia para redes bayesianas, tanto exactos como aproximados [1]. En general, los primeros proporcionan respuestas correctas usando más recursos computacionales, mientras que los otros pueden ser más eficientes a costa de producir respuestas menos precisas. En nuestro trabajo previo probamos ambos tipos (ver tabla 1) y concluimos que, para un tamaño de grafo razonable, el algoritmo del árbol de uniones [11] (junction tree, en inglés) obtenía el mejor rendimiento en términos de recursos computacionales. Su complejidad es exponencial en el ancho de árbol $w$ del árbol de uniones (una estructura secundaria obtenida a partir de la red bayesiana del modelo) y también depende del número de nodos, $n$. En este trabajo también empleamos el mismo método.

Tabla 1: Métodos comunes en inferencia bayesiana

\begin{tabular}{|c|c|c|}
\hline Método & Complejidad & Tipo \\
\hline Árbol de uniones & $O(n \cdot \exp (w))$ & Exacto \\
\hline Eliminación de variables & $O\left(n^{2} \cdot \exp (w)\right)$ & Exacto \\
\hline Propagación de creencia iterativo & Any-time & Aproximado \\
\hline Ponderación de probabilidad & Any-time & Aproximado \\
\hline
\end{tabular}

Nuestra solución utiliza un elemento básico llamado sensor bayesiano, modelado mediante una red bayesiana, que no solamente representa un sensor real, sino también información adicional que permite el diagnóstico inteligente y la mejora sensorial. La figura 1 muestra la estructura de este sensor bayesiano genérico, formado por tres subredes diferentes (rectángulos) y un nodo multiplexor (elipse) que explicamos a continuación.

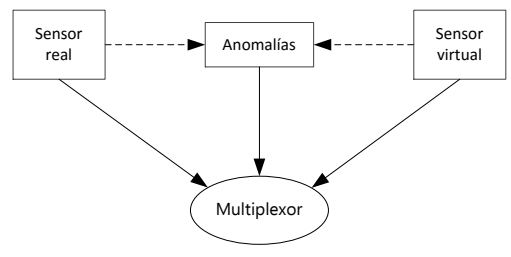

Figura 1: Sensor genérico basado en redes bayesianas. Se puede construir una arquitectura sensorial bayesiana completa interconectando elementos como éste a través de sus multiplexores.

La subred sensor real representa un sensor existente a bordo del robot móvil, pero también puede emplearse para modelas otros tipos de información sensorial primaria. Esta subred contendrá un nodo cuyos valores representan las medidas del sensor. La subred sensor virtual recibe información de 
otros sensores bayesianos (directamente o a través de cálculos) para emular el comportamiento de su sensor real correspondiente cuando éste falla, y se utiliza para deducir sus datos, es decir, para recuperación. La subred anomalías indica si existe una limitación o fallo en el sensor asociado. Esto puede deducirse, por ejemplo, utilizando información de otros sensores que puedan tener alguna influencia en el funcionamiento del sensor asociado. Si no existe tal relación, es posible obtener información de anomalías mediante la comparación de las subredes real y virtual. Ambas alternativas son posibles pero no obligatorias (esto se representa con línea discontinua en la figura 1). Finalmente, el nodo multiplexor selecciona la medida inferida para el sensor (real o virtual) más apropiada de acuerdo a la información de anomalías. Si hay una alta probabilidad de fallo, entonces el sensor virtual tendrá mayor influencia que el real en el resultado final. El nodo multiplexor es una variable discreta con los mismos valores que los de los nodos terminales de las subredes sensor virtual y real.

\section{INSTANCIACIÓN PARA UN ROBOT MÓVIL}

En este trabajo hemos empleado el robot CRUMB de la Universidad de Málaga [10] para implementar la solución definida anteriormente (ver figura 2). CRUMB es un robot Turtlebot con una plataforma móvil Kobuki y una batería de sensores: tres detectores de colisión, dos codificadores magnéticos, tres detectores de precipicio, un giroscopio, dos sensores de levantamiento de rueda, un sensor de visión RGB-D Kinect y un telémetro láser 2D Hokuyo. Aquí nos centraremos en el láser para probar nuestra solución, pero también utilizaremos la cámara RGB-D así como los detectores de colisión, los codificadores e información sobre el entorno.

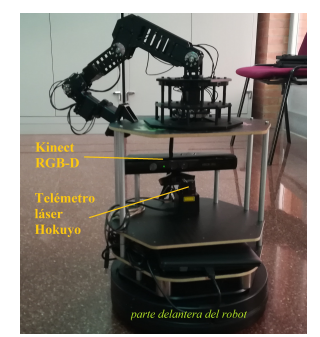

Figura 2: El robot CRUMB y los principales sensores utilizados en este trabajo.

La subred sensor real contiene tantos nodos como elementos se deseen representar del vector de medidas que proporciona el telémetro láser 2D (es decir, rayos). Cada nodo es una variable aleatoria con una discretización adecuada de las medidas de distancia. El nodo multiplexor es replicado para cada rayo láser y representa las distribuciones de probabilidad finales que deseamos obtener. La estructura es trivial a partir de la figura 1. En la subred de anomalías empleamos información del entorno así como datos de otros sensores para detectar fallos en el telémetro láser. El ruido es un problema común en este tipo de sensores; además, un rayo láser puede reflejarse en una superficie especular o equivalente, de manera que la distancia resultante es mucho mayor que la real. Estos dos problemas se pueden clasificar como discontinuidades. En nuestra red se detectan comparando el valor de un determinado rayo con la mediana de un grupo de rayos adyacentes.

Las condiciones del entorno son también muy importantes para el correcto funcionamiento de este sensor. Por ejemplo, una mala iluminación en interiores puede producir medidas erróneas, así como la presencia de lluvia en exteriores y también entornos con temperaturas muy elevadas. Todos estos problemas se han tenido en cuenta en nuesto modelo considerando sensores de iluminación, temperatura y humedad, pero también información sobre el día, la hora y la localización geográfica del robot (ver figura 3) ya que estos datos pueden proporcionar información similar a través de Internet. Se considera que las anomalías de tipo ambiental afectan todos los rayos láser por igual, por lo que los nodos de esta subred solamente se usarán una vez en la red completa.

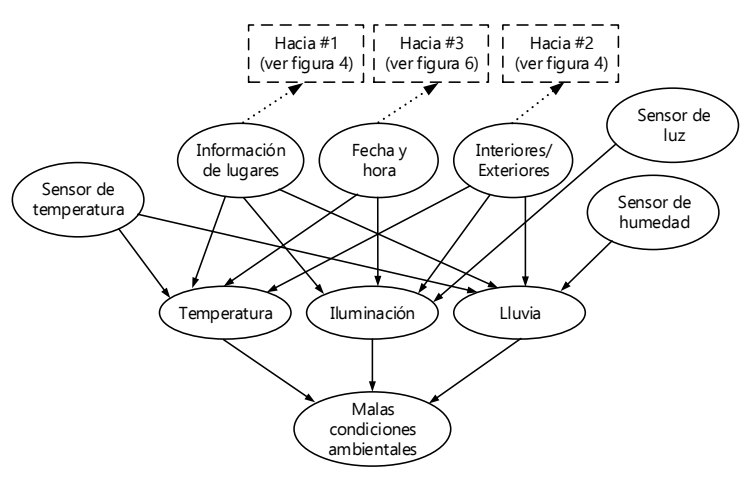

Figura 3: Detección de malas condiciones ambientales para la subred de anomalías.

Asimismo, existen errores sistemáticos que afectan a un telémetro láser, como la detección de objetos demasiado esbeltos, transparentes o negros. Para esta parte de la subred de anomalías empleamos un sensor de distancia alternativo (la información de profundidad que proporciona la cámara Kinect), una imagen RGB y algunos nodos de la parte ambiental (ver figura 4). Aquí, los valores reales del telémetro láser se comparan con los del sensor de distancia alternativo; si hay una di- 
ferencia significativa, entonces se habrá detectado una anomalía. Podemos distinguir la anomalía de radiación absorbida (objetos negros) combinando la información del lugar en el que se encuentra el robot y el porcentaje de píxeles negros en la imagen RGB; la anomalías de objeto no detectado (obstáculos demasiado esbeltos o transparentes en la trayectoria de los rayos láser) tiene en cuenta el sentido de la diferencia entre la medida del láser y la de los sensores alternativos y también la lista sobre información de los lugares (por ejemplo, si sabemos que nos encontramos en un lugar con objetos transparentes, como ventanas, y uno o más sensores alternativos indican distancias más cortas que el láser, la probabilidad de fallo por esta razón será elevada). Si hay una diferencia importante entre las distancias medidas y las anomalías mencionadas no son ciertas, existe un nodo (fallo por error de lectura) que aumenta su probabilidad. Dado que todos los rayos están afectados por estas anomalías, esta parte de la red se copia para cada rayo. Sin embargo, podemos no necesitar tantos nodos para la detección de píxeles negros, por lo que podemos definir el número de estos nodos mediante la especificación de una cierta tasa.

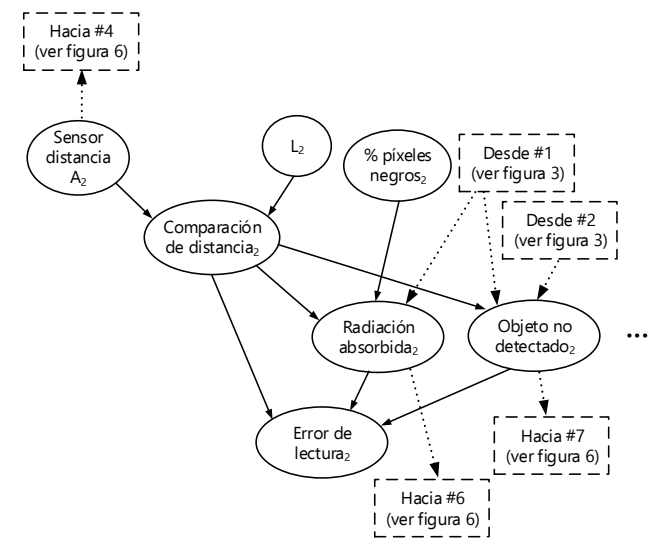

Figura 4: Detección de errores sistemáticos para la subred de anomalías correspondiente al rayo \#2 del telémetro láser.

Todas las anomalías descritas se resumen en un nodo terminal que indica si finalmente un determinado rayo tiene un estado erróneo. Este modelo se muestra en la figura 5. La subred virtual propuesta (véase figura 6) tiene en cuenta el valor de los sensores de colisión y la velocidad lineal de la plataforma bajo un cierto ángulo de barrido del láser, además de algunas anomalías. Suponiendo navegación reactiva, si la velocidad lineal es reducida, la probabilidad de hallar un obstáculo cercano será alta. Por otro lado, el nodo de colisión obtiene información sobre las mismas, que, en caso de ocurrir, implicarán una alta probabilidad de distancia corta. No es necesario que cada rayo de- ba considerar la información de colisión o velocidad de forma independiente, ya que dicha información afecta por igual a muchos rayos adyacentes. Por ello, la implementación procedimental permite definir estos nodos a una cierta proporción.

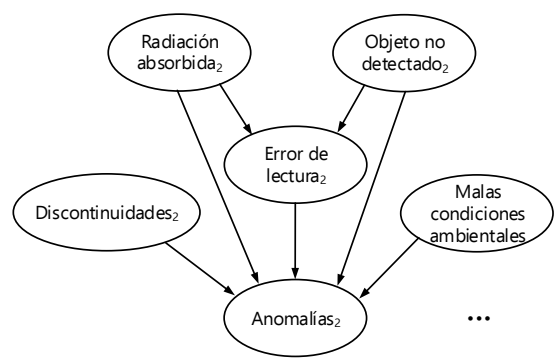

Figura 5: Integración de anomalías para el rayo \#2 del telémetro láser. Los nodos que aquí aparecen son los mismos que los descritos en la figura 4 .

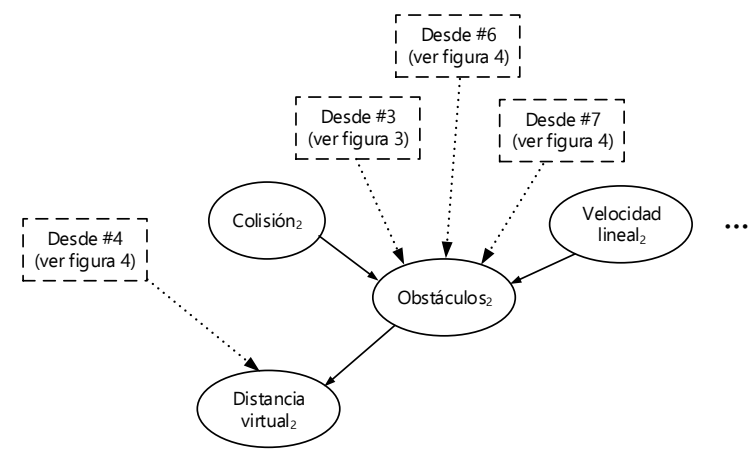

Figura 6: Subred sensor virtual para el rayo \#2 del telémetro láser.

Esta subred también emplea conocimiento procedente de la de anomalías sobre la presencia de un objeto que no ha sido detectado (demasiado esbelto, transparente o negro). Además, la información acerca de la fecha y la hora es importante para estimar la cantidad de personas u otro tipo de obstáculos móviles presentes en la escena, por lo que esta subred también utiliza conocimiento externo. La subred virtual se repite para cada rayo láser, dado que es esencial obtener una estimación de la distancia para poder compararla con los datos reales procedentes del láser.

\section{EVALUACIÓN EXPERIMENTAL}

La solución descrita anteriormente se ha evaluado en el robot móvil CRUMB en varios experimentos que se describen en este apartado. El robot cuenta con un PC portátil a bordo dotado con un Intel Celeron N2840 a $2.16 \mathrm{GHz}$ y $2 \mathrm{~GB}$ DDR3 que ejecuta Ubuntu 14.04 con ROS [12]. Dado que no estamos interesados en tratar limitaciones computacionales en este trabajo, hemos 
utilizado otro PC para ejecutar de forma remota nuestro software de inferencia bayesiana, que cuenta con un Intel Core i3 $3217 \mathrm{U}$ a $1.8 \mathrm{GHz}$ y 6 GB DDR3 y que ejecuta Ubuntu 16.04 con ROS. Nuestro software de inferencia bayesiana junto con el modelo descrito en el apartado 3 se ha implementado en MATLAB empleando la Bayes Net Toolbox (BNT) [13]. La red bayesiana se ha creado con 10 rayos láser (representados por 10 nodos multiplexores en la red, que corresponden, aproximadamente, a 60 grados de campo de visión o fov) con 10 posibles distancias para cada uno, las cuales van desde 1 (obstáculo no detectado) hasta 10 (máxima distancia). La red resultante tiene 148 nodos. En cada experimento se ha ejecutado un bucle de control en el que primero se recogen datos sensoriales, depués se evalúa la arquitectura bayesiana con estas evidencias y finalmente se envían comandos de velocidad al robot si es necesario.

Nuestro primer experimento muestra la situación de anomalía que se produce a causa de la presencia de objetos negros en la escena, los cuales absorben la radiación procedente de los rayos láser. En la figura 7(a) podemos ver el diseño de este experimento; en la figura 8 se muestran los resultados de inferencia. En esta prueba el robot no se mueve.

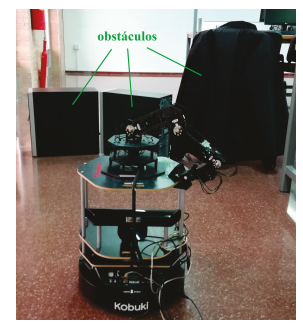

(a) Anomalía por objetos negros

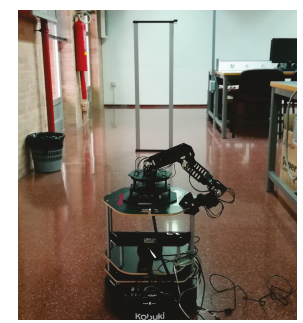

(b) Anomalía por objetos esbeltos
Figura 7: Diseños experimentales para (a) anomalía por obstáculos negros y (b) por obstáculos esbeltos. En la última, centramos ambos sensores de distancia en el lado derecho del marco metálico.

Como podemos observar en la figura 8, la presencia de objetos negros afecta a todos los rayos, pero muchos de ellos proporcionan la medida correcta dado que ambos sensores reales detectan la distancia adecuadamente. Nótese que el telémetro láser no percibe obstáculo en el cuarto rayo; por el contrario, la cámara Kinect sí lo detecta. Los resultados de inferencia muestran una alta probabilidad de fallo a causa de la anomalía por radiación abrorbida, como en otros rayos, pero el sistema finalmente asigna la distancia correcta al nodo multiplexor correspondiente. En este caso, la cámara Kinect se considera sensor de apoyo (alternativo).
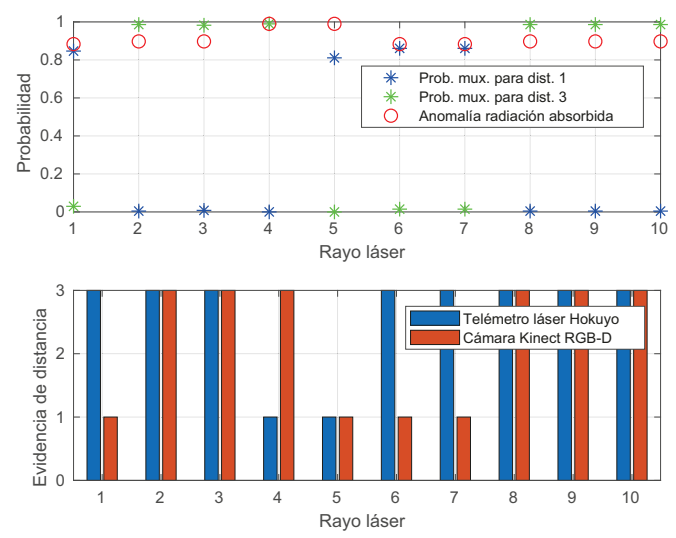

Figura 8: Resultados de inferencia para la prueba de anomalía de obstáculos negros. Se representan todos los rayos (horizontal) así como los datos de evidencia de los sensores reales (figura inferior) donde 1 corresponde a obstáculo no detectado y $2,3 \ldots$ a obstáculos a distancias crecientes.

El segundo experimento expone el robot CRUMB a malas condiciones de iluminación. Hemos utilizado iluminación incandescente como se muestra en la figura 9 para emitir radiación infrarroja en la misma banda de frecuencias que la de los rayos láser. Esto provoca una corrupción de datos significativa en ambos sensores de distancia.

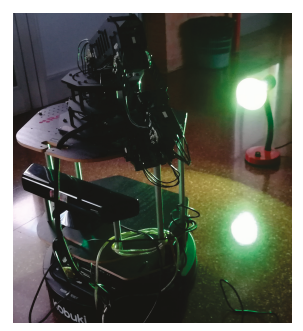

Figura 9: Diseño experimental para la prueba de anomalías por malas condiciones de iluminación. La bombilla queda situada justamente en frente de los sensores.

La figura 10 muestra los resultados obtenidos cuando se emplean condiciones de iluminación extremas, de manera que ambos sensores de distancia resultan seriamente afectados. En este caso, el sistema de inferencia asigna una probabilidad alta a la anomalía por mala iluminación a todos los rayos.

En el tercer y último experimento mostramos cómo trata nuestra solución las anomalías producidas por la presencia de objetos esbeltos (ver figura 7(b)). El telémetro láser no puede detectar ningún obstáculo debido a dos razones: dicho obstáculo es demasiado delgado y la pared se encuentra, a su vez, fuera del rango de medida. Hemos utilizado aquí 10 rayos láser, como es habi- 

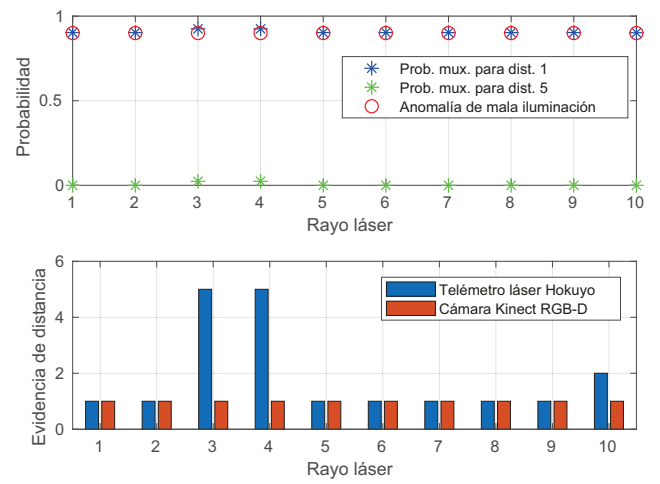

Figura 10: Resultados de inferencia para la prueba de anomalía por malas condiciones de iluminación. Se representan todos los rayos. Como recordatorio, la distancia 1 implica obstáculo no detectado, mientras que 5 corresponde a un rango discretizado de distancia de entre $3.3 \mathrm{~m}$ a $4.4 \mathrm{~m}$.

tual, pero esta vez concentrados en una región del vector de medidas reales para detectar con mayor precisión el lado derecho del obstáculo esbelto. Los resultados experimentales (figura 11) muestran que la distancia real se recupera para ambas anomalías.
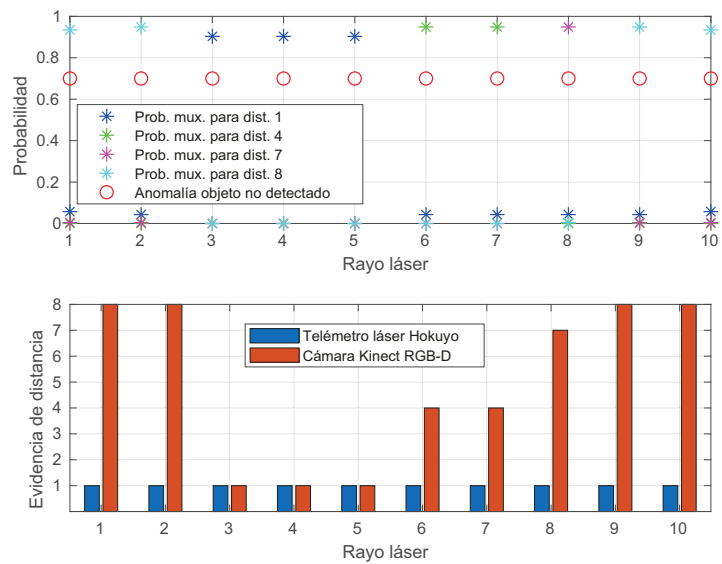

Figura 11: Resultados de inferencia para la prueba de anomalías por obstáculos esbeltos. El obstáculo se detecta mediante una distribución espacial más fina de los rayos láser. De nuevo, la distancia 1 significa que no se ha detectado obstáculo.

Los rayos \#6 y \#7 impactan sobre el obstáculo, que está situado a 2.7 metros del robot. El sistema de inferencia ha asignado una alta probabilidad a la distancia correcta (la número 4, que representa un rango de 2.2 a 3.3 metros) y el resto de los rayos impactan sobre la pared (correctamente asignados a las distancias 7 y 8). Sin embargo, los rayos desde el \#3 al \#5 no se han podido recuperar, ya que su distancia no está disponible en ningún sensor.
Aunque el tema principal de este artículo no sea la eficiencia computacional del algoritmo del árbol de uniones, también se han calculado los tiempos de ejecución para cada experimento, incluyendo la adquisición de datos sensoriales, el cálculo de evidencias (en los casos en que dichas evidencias no sean triviales a partir de los datos sensoriales directos) y la ejecución del método de inferencia bayesiana. Los resultados se resumen en la figura 12. El tiempo medio de ciclo es $1.3981 \mathrm{~s}$ y el tiempo medio de inferencia es $0.3348 \mathrm{~s}$.

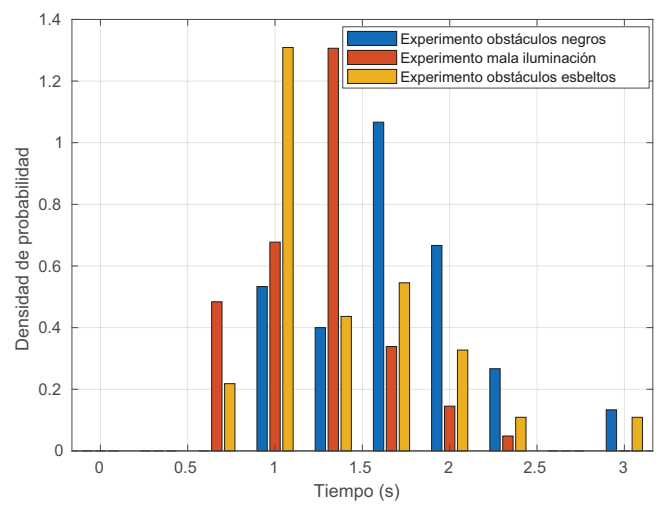

Figura 12: Distribuciones de probabilidad de los tiempos de ejecución para una iteración del bucle de control en todos los experimentos.

\section{CONCLUSIONES}

En este trabajo hemos mostrado cómo un novedoso marco basado en inferencia bayesiana puede constituir una base adecuada para el desarrollo de sensores virtuales de una forma rigurosa. Dado que las posibilidades de los sensores bayesianos virtuales son numerosas y cubren un amplio rango de aplicaciones, nos hemos centrado aquí en su uso para el diagnóstico de sensores de robots móviles y la recuperación de datos. Nuestros experimentos ilustran las capacidades de la solución sensorial bayesiana para inferir fallos en los sensores y las causas de estos fallos, para recuperar los datos sensoriales incluso en dichas situaciones de anomalía, así como el empleo, para todos ellas, de conocimiento proporcionado por un humano (de sentido común, representado en la estructura de la red) y de fuentes externas (ambiental).

En el futuro existen una serie de problemas que planeamos abordar: en primer lugar, el coste del método de inferencia aquí empleado (árbol de uniones) no es adecuado para cualquier tarea robótica, por lo que se necesitan mejoras para reducir dicho coste (por ejemplo, paralelización, empleo de algoritmos aproximados o abstracción de la red); en segundo lugar, la red bayesiana se 
debería crear de una forma más autónoma y automática, pero manteniendo la capacidad de reflejar conocimiento humano, por lo que deben desarrollarse procedimientos interactivos para integrar tal conocimiento que permitan descubrir la estructura más probable de la red; finalmente, la aplicación de nuestro enfoque a sistemas sensoriales mucho más sofisticados (por ejemplo, de visión por computador) debería abordarse una vez que el problema de coste computacional esté razonablemente resuelto.

\section{Agradecimientos}

Este trabajo ha sido subvencionado por el Ministerio de Educación a través de la ayuda con código FPU16/02243, por la Universidad de Málaga a través de su plan propio de investigación y el Campus Internacional de Excelencia Andalucía Tech, y por el proyecto de investigación nacional DPI201565186-R.

\section{English summary}

\section{TOWARDS LOW-LEVEL DIAG- NOSIS AND RECOVERY OF ROBOTIC SENSORS THROUGH INFERENCE WITH BAYESIAN NETWORKS}

\begin{abstract}
Existing implementations for virtual sensors do not use a common, rigorous mathematical framework. In this work we aim to homogenize the theoretical support of virtual sensors at a low level, i.e., dealing with their raw data directly in such a way that they can be employed for fault diagnosis, data recovery and other functionalities without changing the base paradigm. Bayesian inference provides a general and principled way of addressing this; moreover, it allows us to integrate knowledge from diverse sources (the sensor devices themselves, human commonsense, environmental data, etc.) and could be hybridized with other approaches, such as neural networks or fuzzy logic. Since the potential of this solution is considerably wide, here we focus on the fault diagnosis, data recovery and external knowledge integration functionalities. Our results with a real mobile robot equipped with two rangefinder sensors and also common, simpler devices demonstrate
\end{abstract}

that the framework has many possibilities for improving the sensory system of a mobile robot through high-level reasoning techniques.

Keywords: Bayesian inference, anomaly detection and recovery, virtual sensors

\section{Referencias}

[1] A. Darwiche. Modeling and Reasoning with Bayesian Networks. New York: Cambridge University Press, 2009.

[2] H. Zhou, S. Sakane. Sensor Planning for Mobile Robot Localization-A Hierarchical Approach Using a Bayesian Network and a Particle Filter, IEEE Transactions on Robotics, vol. 24, no. 2,pp. 481-487, April 2008.

[3] D. Song, C. Henrik Ek, K. Huebner, D. Kragic. Task-Based Robot Grasp Planning Using Probabilistic Inference, IEEE Transactions on Robotics, vol. 31, no. 3, pp. 546-561, June 2015 .

[4] E. Besada-Portas, J. A. Lopez-Orozco, J. M. de la Cruz. Unified fusion system based on Bayesian networks for autonomous mobile robots, Proceedings of the Fifth International Conference on Information Fusion, Annapolis, MD, USA, 2002, pp. 873-880, vol.2.

[5] P. Bessière. Probability as an Alternative to Logic for Rational Sensory-Motor Reasoning and Decision, in Probabilistic Reasoning and Decision Making in Sensory-Motor Systems, vol. 46, Berlin: Springer-Verlag, 2008, pp. 318.

[6] B. Cai, L. Huang, M. Xie, Bayesian Networks in Fault Diagnosis. IEEE Transactions on Industrial Informatics, vol. 13, no. 5, pp. 22272240, October 2017.

[7] R. Munguia, A. Grau. Closing Loops With a Virtual Sensor Based on Monocular SLAM, IEEE Transactions on Instrumentation and Measurement, vol. 58, no. 8, pp. 2377-2384, August 2009.

[8] P. Buschka, A. Saffiotti. A virtual sensor for room detection, IEEE/RSJ International Conference on Intelligent Robots and Systems, Lausanne, 2002, pp. 637-642, vol. 1. 
[9] I. Hashlamon, K. Erbatur. Joint sensor fault detection and recovery based on virtual sensor for walking legged robots, IEEE 23rd International Symposium on Industrial Electronics (ISIE), Istanbul, 2014, pp. 1210-1214.

[10] M. Castellano-Quero, I. Fernández-Vega, J.A. Fernández-Madrigal, A. Cruz-Martín. Métodos de inferencia bayesiana empotrados para el diagnóstico y mejora sensoriales de un robot móvil, II Jornadas de Computación Empotrada y Reconfigurable (JCER2017), Málaga, 2017, pp. 667-676

[11] C. Huang, A. Darwiche. Inference in Belief Networks: A Procedural Guide, International Journal of Approximate Reasoning, vol. 15, no. 3, pp. 225-263, October 1996.

[12] M. Quigley, K. Conley, B. Gerkey, J. Faust, T. Foote, J. Leibs, R. Wheeler, A. Ng. ROS: an open-source Robot Operating System, Proc. ICRA Workshop Open Source Software, pp. 1-6, May 2009.

[13] K. P. Murphy. The Bayes Net Toolbox for MATLAB, Computing Science and Statistics, vol. 33, no. 2, pp. 1024-1034, October 2001.

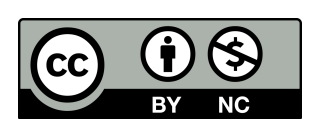

(C) 2018 by the authors. Submitted for possible open access publication under the terms and conditions of the Creative Commons Attribution CC-BY-NC 3.0 license (http://creativecommons.org/licenses/by-nc/3.0/). 